\title{
Formation of Coherent Multi-Element Resonance States in Metamaterials
}

\author{
Elena Semouchkina \\ Michigan Technological University \\ USA
}

\section{Introduction}

Employment of metamaterials in cloaking devices, which could make concealed objects invisible, is based on the capability of resonators used as metamaterial "atoms" to provide effective material parameters, ranging from any positive to any negative values, in the vicinity of the resonance frequency. Metamaterials comprising layers with variously sized resonators (Schurig et al., 2006) or layers with variable density of identical resonators (Semouchkina et al., 2010) have been proposed to obtain the desired spatial distribution of the medium parameters, for example, radial variation of the effective permittivity or permeability in the shell prescribed by the transformation optics relations for cloaking cylindrically shaped objects (Leonhardt, 2006; Pendry et al., 2006). Such approach could be justified, if resonators contribute to the value of the effective parameters by the same way as polarized atoms contribute to the value of the polarization of a dielectric medium, i.e., if the responses of individual atoms are additive so that the medium response exhibits the same dispersion as the response of an individual atom. Then the material can be described by the effective medium theory (EMT), which was adopted for metamaterails description at the onset of metamaterial studies (Pendry et al., 1999; Pendry \& Smith, 2003; Smith \& Pendry, 2004; Smith et al., 2006). According to the EMT, a polar dielectric material could be represented by a set of parallel dipoles switching their directions by $180^{\circ}$ in dependence on the phase of the external field. Resonance modes in the "atoms" of metamaterials could also be represented by equivalent dipoles and, similarly to atoms in dielectrics, these dipoles are expected to respond accordingly, i.e. to follow the phase of the external field and demonstrate a coherent response within the half wavelength of incident radiation. However, observation of the performance of the infrared invisibility cloak designed from glass resonators (Semouchkina et al., 2010) has shown that obtaining a coherent response of the cloak structure presents a serious problem, if resonators in the metamaterail are electromagnetically coupled. The problem of coupling between resonators in metamaterials, however, remains to be largely ignored in the literature.

It is well known that metamaterials, as a rule, are designed as periodic structures of closepacked resonators. When the dimensions of resonators are ten times smaller than the wavelength, the EMT is considered to be applicable, so that the waves "do not see" the atoms and propagate in the metamaterial as in a homogenized, i.e. in a uniform medium. Such homogenization is supposed to make metamaterials essentially different from 
photonic crystals (Pendry \& Smith, 2003). It is worth noting that the lattice parameter was not thought to be critical for the EMT application, so that the only ultimate requirement for metamaterials in this attitude is that the lattice parameter should not be equal to the half wavelength of incident radiation, which is typical for photonic crystals. Therefore, for example, the metamaterial for the microwave cloak operating at $8.5 \mathrm{GHz}$ (Schurig et al., 2006), was composed of split ring resonators (SRR) with the planar dimensions of $3 \mathrm{~mm} \times 3$ $\mathrm{mm}$ packed in concentric arrays with the inter-resonators separation of $0.17 \mathrm{~mm}$, which is negligible compared to the resonator size. Such close packing of SRRs causes questions about possible effects of interaction between resonators and elementary resonance splitting that could deteriorate the EMT applicability.

Our earlier studies (Semouchkina et al., 2004, 2005) indeed pointed out at strong splitting of elementary resonances in close-packed resonator arrays of conventional metamaterials consisting of metal elements. At the modeling of an extended finite metamaterial block, which consisted of 36 SRRs and 12 elongated metal strips arranged in unit cells quite similar to typical arrangements employed in conventional metamaterials, it was shown that at the frequencies corresponding to the metamaterial transmission band, elementary resonances were essentially coupled and integrated in 3D networks, an example of which is presented in Fig. 1. As a sequence of coupling and splitting, most resonators responded resonantly at multiple frequencies and formed specific accociations at different frequencies in the transmission band, making the metamaterial essentially nonuniform with the character of inhomogenities changing in dependence on frequency. No regular wave front propagation across the sample was observed, so that the energy transfer occurred rather due to the hopping mechanism similar to that thought to be inherent in the CROWs (coupled resonator optical waveguides (Yariv et al., 1999).

It is logical to suggest that phenomena similar to the mentioned above should take place in any close-packed metamaterial structures including those used in the microwave cloak described in (Schurig et al., 2006). The reason why these phenomena have not been revealed by the authors can be seen in the employment of simulation models, in which real multiresonator cloak structures were replaced by layered material structures with prescribed values of the effective permeability for each layer.

An additional reason, why coupling phenomena in metamaterials remain underexplored is seen in the typical approach to consider metamaterial properties to be identical to the properties of a single cell and to ignore interaction between cells. This approach is based on the results of unit cell simulations which could not reveal resonance splitting even at small distances between neighboring resonators defined by the dimensions of the unit cell, because of employment of plane wave excitation source at normal wave incidence and periodic boundary conditions (PBC) at the cell boundaries normal to the direction of wave propagation. Such arangement, however, could only model an infinite 2D array of unit cells and not a 3D metamaterial medium. Our results indicate that drastic changes in electromagnetic (EM) metamaterial response could be observed at stacking 2D arrays in the direction of wave propagation to form a 3D structure, because incident wave has a different phase, when encounters each 2D array. Significant changes also occur in finite $2 \mathrm{D}$ arrays and in arrays with some distortions of periodicity that is unavoidable in practice. 

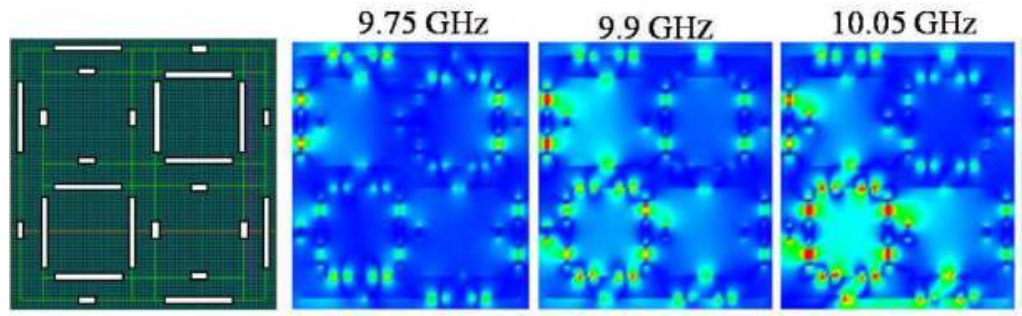

$10.35 \mathrm{GHz}$
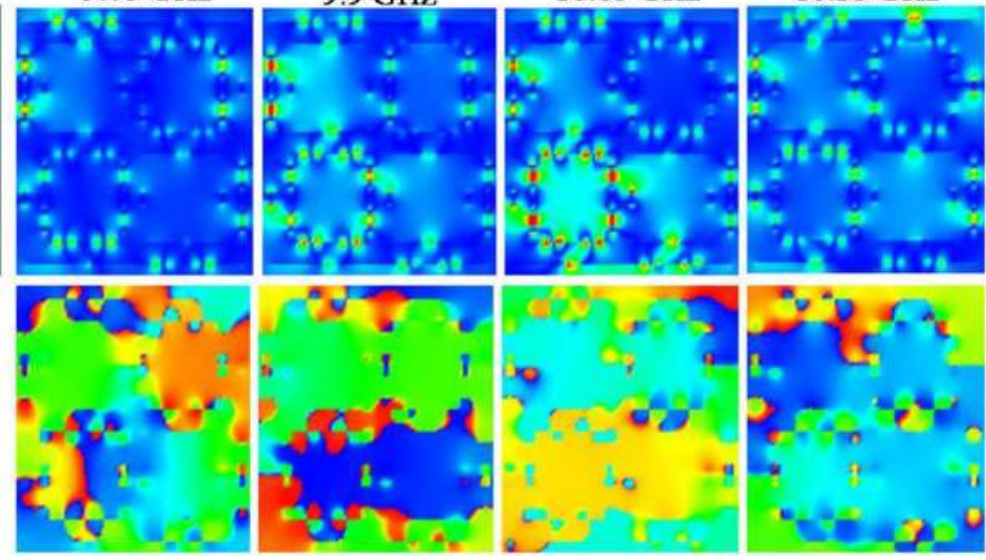

Fig. 1. Cross-section of the metamaterial block depicting SRR sections (longer strips) and cut wires (shorter strips) placed normally to the figure plane (left figure); and distributions of magnetic field amplitude (upper row) and phase (lower row) in the same cross-section at four frequencies within the metamaterial transmission band. Bright spots in the upper row correspond to the areas with highest field magnitude.

This Chapter addresses the listed above underexplored metamaterial problems and describes the effects caused by coupling and splitting of resonances in two types of metamaterial multi-resonator structures, i.e. those composed of SRRs and of dielectric resonators. The results presented here demonstrate that EM responses provided by these structures cannot be reduced to the responses of their constituent single resonators as it is assumed at application of the EMT. The Chapter also shows that splitting of resonances in arrays of dielectric resonators leads to the formation of a bandgap dividing two transmission zones, one at lower, and another one at higher frequncies. While at the gap frequencies elementary resonances in arrays look sporadically flashing at various multiple frequencies, the lower frequency transmission zone is characterized by overlapping of the resonance fields and slow wave propagation. The transmission zone at higher frequencies can be characterized by a coherent response of elementary resonators within halfwavelength distances and by superluminal phase velocities of propagating waves. The obtained results bridge the gap between the properties of metamaterials and photonic crystals employing Mie resonances.

\section{Resonance splitting in metamaterial arrays composed of SRRs}

\subsection{Specific features of SRR characterization}

Investigation of the resonance splitting phenomena in SRR, which is presented in the following sub-sections, has been performed on fragments of SRR arrays quite similar to the arrays used in the design of the microwave invisibility cloak (Schurig et al., 2006). This cloaking shell was formed from concentric arrays of unit cells each including one SRR. The size of the sell was of $3.33 \mathrm{~mm} \times 3.33 \mathrm{~mm}$ in the SRR plane and $3.18 \mathrm{~mm}$ normal to this plane with the SRR located in the central position. The SRRs were placed on the substrate with the 
thickness of 381 microns and the relative permittivity of 2.33. In the simulations described in the next sub-section, the SRRs with identical parameters (Fig. 2a) were used to build arrays of unit cells similar to various fragments of the cloak described in (Schurig et al., 2006). The only difference between the investigated metamaterial fragments and those used in the publication was that fragments in our case were flat. However, as the radiuses of concentric arrays in (Schurig et al., 2006) were relatively big compared to the cell size, flat arrays with the lengths of up to six cells were a good approaximation of fractions of concentric arrays in the cloak metamaterial. The propagation vector (k-vector) of the incident wave in our studies was always directed along the array rows in the plane of the SRR location, while magnetic field was directed normally to the SRR planes. Therefore, the investigated arrays had to perform similarly to the cloak fragments located close to the diameter of the shell normal to the k-vector of the incident wave. First, the SRR responses in air and at the placement of the resonators on the dielectric substrate have been investigated. Numerical simulations were performed for three arrangements: 1) at the plane wave incidence on the object, 2) at the placement of the SRR in the waveguide of standard dimensions with perfect electric conductor (PEC) walls, and 3) at application of waveguide ports to the standard unit cell and/or to the unit cell increased to incorporate the resonance field "halos" (the following section describes how the "halo" dimensions were found).

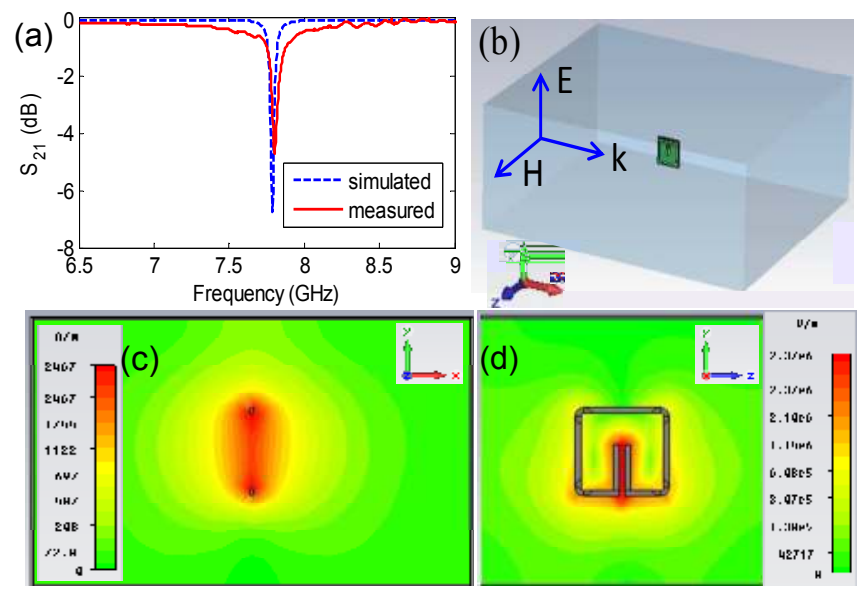

Fig. 2. Resonance in a single SRR placed in the waveguide WR137: (a) measured and simulated transmission spectra $S_{21}$; (b) arrangement for the measurements; (c) magnetic and (d) electric field patterns.

All simulations were conducted by using the commercial full-wave software package CST Microwave Studio. For the two first arrangements the Transient Solver has been used, while for the third one - the Frequency Domain Solver. Numerical experiments with waveguide ports allowed for obtaining the scattering parameter spectra convenient for controlling the resonance frequency $f_{\text {res }}$, while in experiments with plane wave incidence, the resonance was detected by monitoring the signal of $\mathrm{H}$-field probes placed at the expected maximum field locations in the resonators. Three types of SRRs that differed by the lengths of their inner slot forming strips $s$ and the radii of the corners $r$, as summarized in Table 1, have 
been characterized. The values of $s$ and $r$ were taken very close to those characteristic for the SRRs of the $1^{\text {st }}, 2^{\text {nd }}$, and $3^{\text {rd }}$ concentric arrays of the microwave cloak described in (Schurig et al., 2006). Small corrections, however, have been introduced in order to provide fitting of the chosen $s$ and $r$ to the least square dependences which were built to express interrelation between these parameters of SRRs and the effective permeability found in (Schurig et al., 2006) for unit cells with SRRs of proper dimensions.

\begin{tabular}{|c|c|c|c|c|c|c|c|}
\hline \multirow{3}{*}{$\mathrm{N}$} & \multirow[b]{2}{*}{$S$} & \multirow[b]{2}{*}{$r$} & \multirow{3}{*}{ substrate } & \multicolumn{2}{|c|}{ Transient solver } & \multicolumn{2}{|c|}{$\begin{array}{c}\text { Frequency domain } \\
\text { solver }\end{array}$} \\
\hline & & & & $\begin{array}{c}f_{\text {res }} \\
\text { free space }\end{array}$ & $\begin{array}{c}f_{\text {res }} \\
\text { waveguide }\end{array}$ & $\begin{array}{c}f_{\text {res }} \\
\text { unit cell }\end{array}$ & $\begin{array}{c}f_{\text {res }} \\
\text { halo cell }\end{array}$ \\
\hline & $\mathrm{mm}$ & $\mathrm{mm}$ & & $\mathrm{GHz}$ & $\mathrm{GHz}$ & $\mathrm{GHz}$ & $\mathrm{GHz}$ \\
\hline \multirow{2}{*}{1} & \multirow{2}{*}{1.654} & \multirow{2}{*}{0.26} & yes & 7.761 & 7.745 & 7.19 & 7.76 \\
\hline & & & no & 9.16 & 9.119 & 8.53 & 9.17 \\
\hline \multirow{2}{*}{2} & \multirow{2}{*}{1.677} & \multirow{2}{*}{0.254} & yes & 7.73 & 7.67 & 7.125 & 7.73 \\
\hline & & & no & 9.125 & 9.075 & 8.49 & 9.185 \\
\hline \multirow{2}{*}{3} & \multirow{2}{*}{1.716} & \multirow{2}{*}{0.244} & yes & 7.673 & 7.657 & 7.027 & 7.673 \\
\hline & & & no & 9.061 & 9.03 & 8.448 & 9.068 \\
\hline
\end{tabular}

Table 1. Parameters and resonance frequencies of various SRRs

As seen from Table 1, numerical experiments with plane wave incidence and with the standard waveguide gave quite close results for $f_{\text {res }}$, while in experiments with unit cells used in (Schurig et al., 2006) this frequency appeared to be essentially lower both for SRRs placed in air and on a substrate. The resulting S-parameter spectra in the latter simulations appeared distorted in comparison to the spectra obtained in the standard waveguide. Similar distortions of resonances are known to be observed at too close placement of metal objects or the domain boundaries to the resonator. Therefore, the simulations have been repeated for computational volumes of increased dimensions. Gradual increase of the volume caused better and better fitting of the simulation results to the data obtained in two other sets of numerical experiments. Saturation of changes and coincidence of the data was observed at the distances between resonator and domain boundaries exceeding $1.833 \mathrm{~mm}$ in the SRR plane and $4.183 \mathrm{~mm}$ in the normal direction. Apparently these dimensions characterize the "halo" of the resonance fields around the SRRs, illustrated by the field patterns presented in Figs. 2a and 2c.

It is obvious that the space occupied by the SRR field "halo" substantially exceeds the volume of unit cells used in (Schurig et al., 2006). As seen in Figs. 2a and 2c, the resonance magnetic and electric fields in the "halo" are not vanishing at the distances from the SRR 
corresponding to the boundaries of the used unit cells, therefore, the resonance formation and the resonance frequencies $f_{\text {res }}$ were affected in respective simulations of such unit cells. In comparison, field magnitudes at the boundaries of unit cells with the size equal to the "halo" size were found to be ten times smaller than those of the cells used in (Schurig et al., 2006) that explains the consistency of the data obtained for the halo-sized cells and the results of other numerical experiments. Only at building the shell of such cells, i.e. at the placement of resonators in arrays of the metamaterial at distances, when "halos" are not overlapped, interaction between resonators could be omitted from consideration. In contrast, the distance between resonators in concentric arrays in (Schurig et al., 2006) did not exceed $0.333 \mathrm{~mm}$, while the distance between arrays (i.e. along the normal to the plane of resonators) was only $3.183 \mathrm{~mm}$.

The data presented in Table 1 do not confirm the expectations for $f_{\text {res }}$ to be of about $8.5 \mathrm{GHz}$ as reported in (Schurig et al., 2006) for both the resonance frequency of basic SRRs and the frequency of the cloak response. Resonances at frequencies close to $8.5 \mathrm{GHz}$ were obtained in our experiments only for the unit cells used in (Schurig et al., 2006) at the placement of SRRs in air. It might be that the dielectric substrate was omitted in models used for simulations in (Schurig et al., 2006). The obtained discrepancy does not seem to be critical for the studies of the splitting phenomena in SRR-based metamaterials employed in (Schurig et al., 2006), although it should be taken into account at verification of the cloaking effect, since this effect should not be expected at $8.5 \mathrm{GHz}$, if the metamaterial perform in accordance to the EMT predictions.

\subsection{Resonances in SRR arrays representing fragments of the microwave cloak}

In order to additionally verify the results of simulations, individual SRRs and various multielement arrays of SRRs with the same dimensions as in (Schurig et al., 2006) have been fabricated, and their EM response has been measured in the waveguide WR137 operating at the single $\mathrm{TE}_{10}$ mode in the range (5-10) GHz. In experiments, the waveguide WR137 loaded with SRR arrays was connected to the PNA-L Network Analyzer N5230A to measure the transmission spectrum. In order to avoid EM interaction between the arrays and waveguide walls, resonators or arrays were supported by a styrofoam layer inserted into the center region of the waveguide. As seen from Fig. $2 \mathrm{~d}$, the resonance in a single resonator placed in the waveguide was observed at $\mathrm{f}=7.78 \mathrm{GHz}$ and not at $8.5 \mathrm{GHz}$, i.e. quite close to the results of our simulations for the resonator with the same dimensions.

Next, linear arrays of SRRs arranged along the direction of k-vector of the incident wave have been investigated. Fig. 3a shows that the arrays of 3-5 SRRs demonstrate strong splitting of the resonance, which increases with the number of resonators in the array. It is interesting to note, that instead of multiple dips in the transmission spectrum correlated with the quantity of resonators in the array, only two well expressed drops in the transmission spectra have been observed. The first drop is accompanied by the coherent response of resonators (Fig. 3b), while at the higher frequency drop the resonators in the line respond with opposite phases in two halves of the array. The phase patterns of the wave propagating in free space inside the waveguide at both resonances show strong disturbances of the wave transmission through the waveguide (the curve between bright and dark parts of the image divides the areas corresponding to the half wavelengths of the propagating waves). 


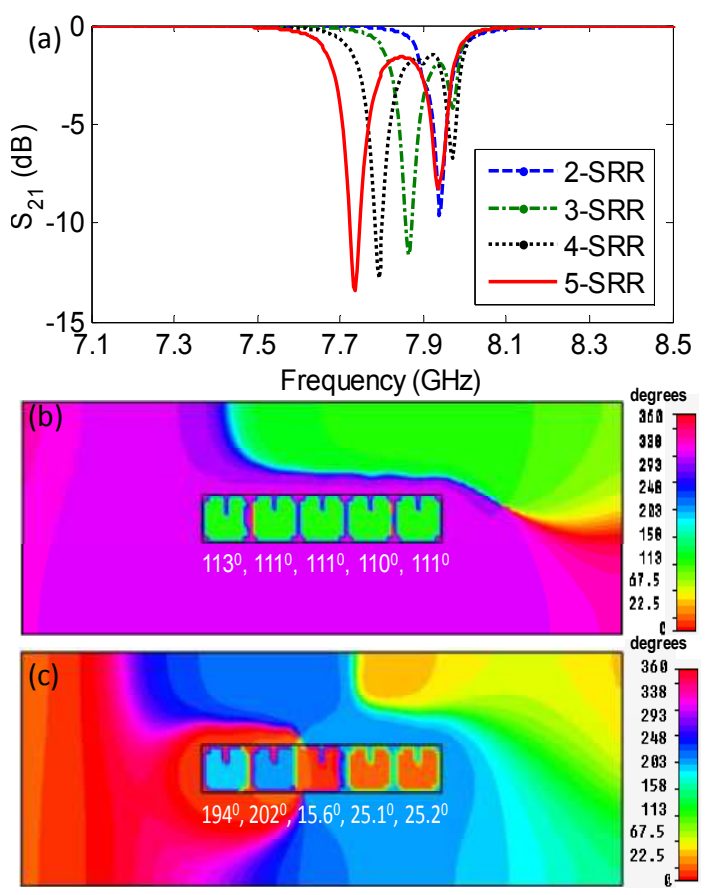

Fig. 3. Resonances in linear arrays arranged in wave propagation direction in the waveguide WR137: (a) $\mathrm{S}_{21}$; (b) coherent response of a 5-SRR array at $7.73 \mathrm{GHz}$; and (c) assymmetric response at $7.95 \mathrm{GHz}$.

Much stronger splitting of SRR resonances has been revealed in the SRR columns (Fig. 4) similar to those used in concentric arrays of the SRRs in (Schurig et al., 2006). Placement of such columns in the waveguide WR137 caused some concerns, since at about $1 \mathrm{~cm}$ height of the columns their resonance fields could interact with the waveguide top and bottom walls that could cause deterioration of the resonances. Therefore, the simulations for these columns were initially performed in free space at plane wave incidence. As such simulations did not provide an opportunity for deriving the transmission spectra, the resonance field in the columns was monitored by using $\mathrm{H}$-field probes placed in geometrically identical points of the SRR cross-sections (Fig. 4a). As seen from Fig.4e the probes revealed three resonances covering more than $1 \mathrm{GHz}$ of the frequency range (i.e. almost five times wider resonance band than that observed for the linear arrays of SRRs arranged along the direction of the incident wave $\mathrm{k}$-vector). It is worth mentioning that each resonator in the column responded resonantly at all split frequencies while the integrated modes at these frequencies were quite different. As seen from Fig. $4 \mathrm{~b}$, at the lower frequency resonance sin-phase oscillations were observed in the two upper resonators, while the third resonator oscillated with a smaller magnitude and with opposite phase. At the higher frequency resonance, the two lower resonators demonstrated sin-phase oscillations while the third resonator oscillated with opposite phase (Fig. 4d). Resonance at the median frequency was supported mainly by sin-phase oscillations in the upper and the lower resonators of the column (Fig. 4c). The third resonator located in the middle of the column did not show strong 
oscillations, however, the phase of these oscillations was shifted by $180^{\circ}$ with respect to the oscillations of two other SRRs.

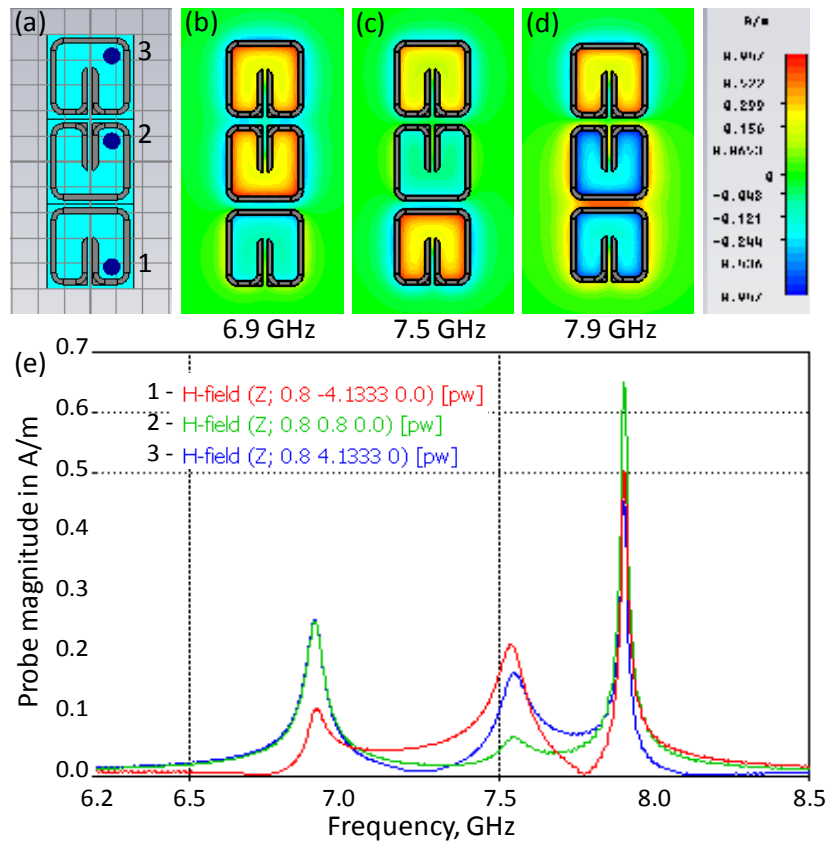

Fig. 4. (a) The model of the SRR column with H-field probes; (b)-(d) H-field resonance responses of the column at signal peak frequencies (colour intensity shows the magnitude of resonance; and (e) the spectra of probe signals.

It is interesting to note that shorted columns of 2 SRRs placed in either "face-to-face" or "back-to-back" arrangement did not demonstrate any splitting of the resonances. As seen from Fig. 5, the former arrangement provided for the unsplit resonance at almost the same frequency as the lower frequency resonance in the column of 3 SRRs, while the latter arrangement provided for the resonance at the frequency close to the frequency of upper resonance in the column of 3 SRRs. It means that the listed above resonances in a 3-SRR column are apparently related to integrated coherent resonances in couples of oppositely arranged SRRs. The coherentness is evident from the phase patterns presented in Fig. 5 on the right.

Essentially more complicated response with multiple resonances of various $Q$ factors was demonstrated by arrays composed of several columns as, for example, the presented by the insert in Fig 6a array of 5 columns. Despite of the complexity, however, it was possible to distinguish three main groups of resonances comparable to resonances revealed in one SRR column (Fig. 4). In fact, the lower frequency group in the $3 \times 5$ SRR array demontsrated coherent resonances in upper rows of the array (Fig. 6b), while the higher frequency group demontsrated coherent resonances in the lower rows of the array (Fig. 6d). The group of resonances at the median frequencies could be characterized by a symmetry of DR 
responses with respect to the central row i.e. the resonators located in upper and lower rows responded coherently, even though neighbouring columns demonstrated opposite phases of oscillations. So, it could be said that every other column responded coherently (Fig. 6c).
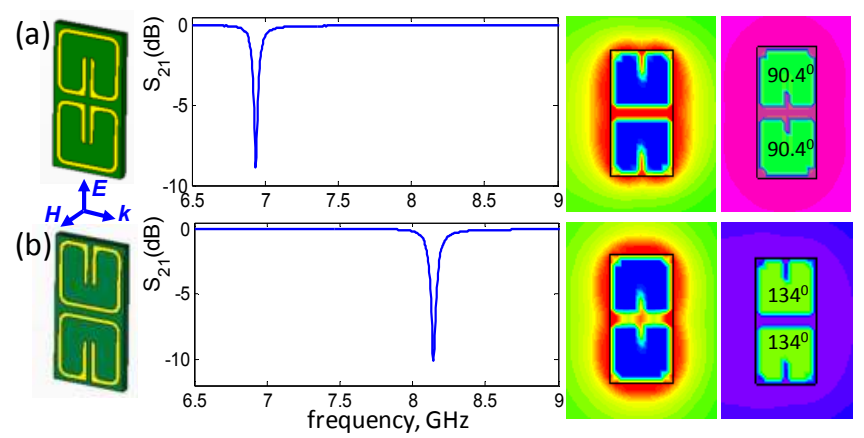

Fig. 5. Geometry, $S_{21}$ spectra, and patterns of H-field magnitude ( $3^{\mathrm{d}}$ column) and phase (4th column) for SRR pairs arranged: (a) "face-to-face" and (b) "back-to-back".

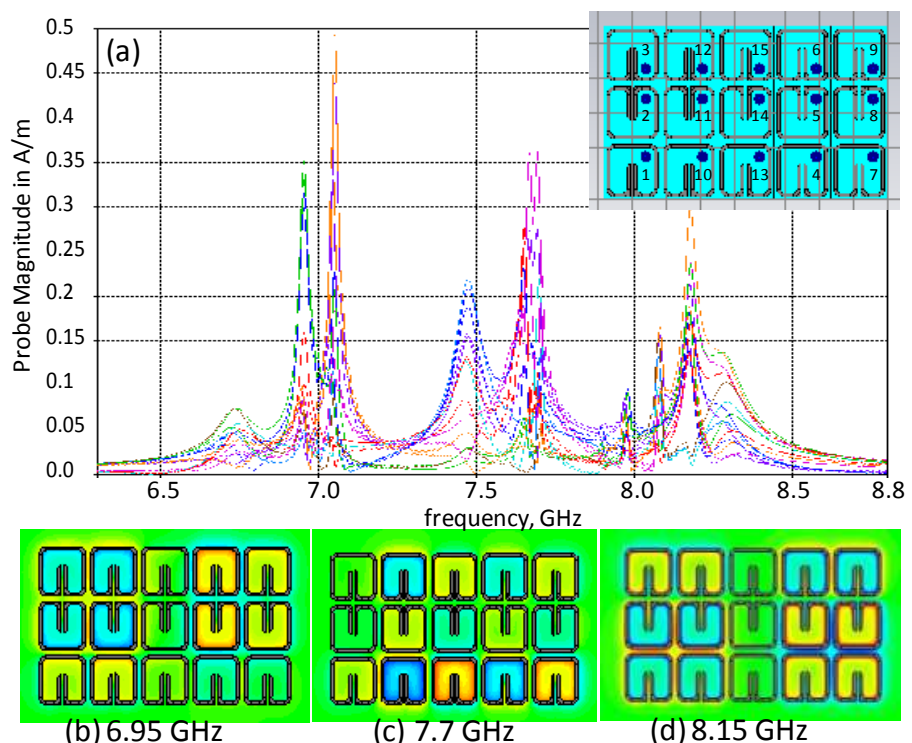

Fig. 6. (a) The spectra of the probe signals; inset shows the SRR array and the H-field probe locations; (b)-(d) typical patterns of the resonance oscillations in the array sampled at 6.64, 7.4 and $8.15 \mathrm{GHz}$ (colour intensity shows the magnitude of resonance oscillations).

Despite of the above mentioned concerns about possible interaction between multi-element arrays and waveguide walls at the measurements we have performed comparison of simulation and measurement results for the transmission spectra at the placement of the $3 \mathrm{x}$ 5 arrays of SRRs in the waveguide WR137. A good matching of the results presented in Fig. 7 is obvious, although both $S_{21}$ spectra demostrate a smaller quantity of the transmission 
dips compared to that observed in the probe signal spectra (Fig. 7). The three groups of resonances, however, could be clearly distinguished in both spectra presented in Fig. 7.

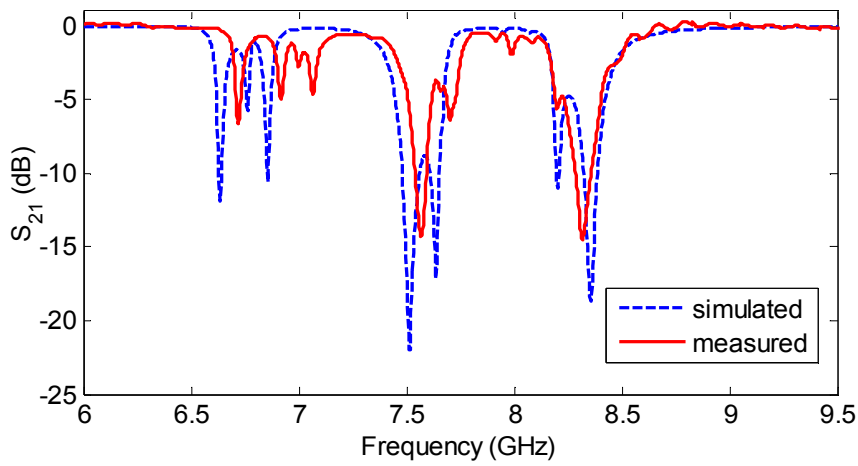

Fig. 7. $S_{21}$ spectra for the $3 \times 5$ array of SRRs in the waveguide WR137: dashed curve simulation results, solid curve - the results of measurements.

The responses of multi-layer structures composed of several planar arrays were found to loose the perspicuity of the splitting phenomena observed in the multicolumn arrays. As seen in Fig. 8c, which presents the probe signal spectra for a three-layer array of SRRs (Fig. $8 \mathrm{a})$, it is difficult to distinguish well separated in frequency groups of resonances similar to those observed for $3 \times 5$ arrays. Instead, split resonances are observed in the essentially extended frequency range of more than $2 \mathrm{GHz}$. Such character of the multi-layer array response makes it quite doubtful to expect that the proposed in (Schurig et al. 2006) design of cylindrical cloak based on close-packed arrays of SRRs would provide the cloaking effect at the frequency determined from the analysis of the response of a single resonator. The reason for obtaining the invisibility effect in (Schurig et al., 2006) should be rather searched for in some specifics of split resonances, which need to be studied additionally. It is worth noting that some of split modes indeed demonstrated a possibility to support the desired coherent response of SRRs across extended areas of investigated arrays as it is shown in Fig. $8 \mathrm{~b}$. It is reasonable to expect that at respective frequencies the participating resonators could contribute to obtaining the desired dispersion of the effective parameters precribed by the transformation optics relations.

\section{Resonances in metamaterial arrays composed of dielectric resonators}

\subsection{Specifics of resonances in dielectric resonators and potential of all-dielectric metamaterials}

Dielectric resonators (DRs), especially those of cylindrical shape, are capable of providing EM response quite similar to that of SRRs. In fact, when the incident wave propagates normally to the axis of the cylinder with its $\mathrm{H}$-field directed along this axis, the DR can support the $\mathrm{TE}_{01 \delta}$ resonance mode, which is equivalent to the formation of a magnetic dipole along the axis of the cylinder, i.e normal to the plane, in which the cross-section of the DR is a circle (Kajfez \& Guillon, 1998). The latter plane should host circular displacement currents comparable to circular currents in SRRs. 


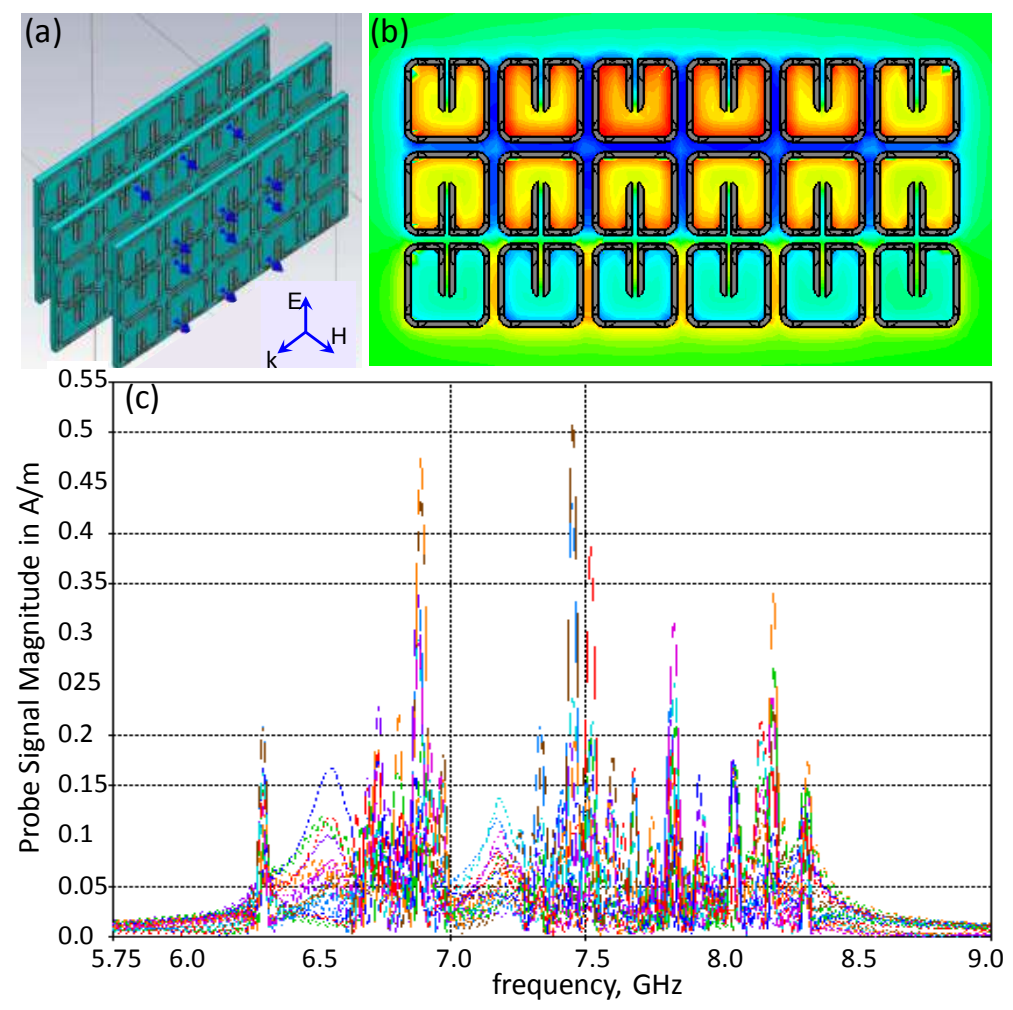

Fig. 8. (a) Geometry of a three- layer SRR array with marked probe locations; (b) coherent response of SRRs at $8.35 \mathrm{GHz}$; and (c) spectra of probe signals revealing enhanced splitting caused by interaction between SRRs in neighbouring layers.

The publication (Semouchkina et al., 2004) was among the first that started the development of all-dielectric metamaterials. The data presented in Fig. 9 confirm the possibility to use dielectric cylinders of a proper size (the diameter of $6 \mathrm{~mm}$ and the height of $3 \mathrm{~mm}$, in this case) and permittivity (dielectric constant of 37.2 in this case) to replace SRRs as magnetic components in metamaterials supporting Lorentz-type resonance necessary for obtaining the effective negative permeability of the medium at microwave frequencies. It was later shown that dielectric resonators can also replace cut metal wires used in conventional metamaterials below their plasma frequency as electric components providing for the effective negative permittivity. Therefore, it was predicted that materials composed of two types of dielectric resonators providing one for electric and another one - for magnetic response could exhibit negative index of refraction (Jylha et al., 2006; Vendik \& Gashinova, 2004). In the work (Semouchkina et al., 2005) it was demonstrated that negative refraction can also be provided by dielectric arrays composed of resonators of one type at the placement of the array on a ground plane. Similar effects in arrays composed of DRs of one type with ground planes or in waveguides have been also reported in (Ueda et al., 2007, 2010). 
Recently, a metamaterial composed of dielectric resonators has been employed to design an invisisbility cloak for the infrared range (Semouchkina et al., 2009, 2010). In difference from earlier works (Cui et al., 2009, Gaillot et al., 2008, Schurig et al., 2006) on microwave and THz cloaks, the design proposed in (Semouchkina et al., 2009, 2010) utilized identical resonators in the entire cloakling shell. Such design does not require to solve technological problems of fabricating concentric arrays of various nano-sized elements, as well as does not demand a wide band of incident light to excite differently sized resonators. In addition, employment of chalcogenide glass as a resonator material should decrease the loss related limitations for the size of concealed objects recently formulated in (Zhang et al., 2009). The estimates based on the level of the extinction coefficient at 1 micron wavelength of incident light gave more than an order less value for the loss tangent of the glass cloak than values characteristic for previously developed cloaks. The required by the transformation optics dispersion of the effective permeability in the novel cloak design was obtained by using a controlled decrease of the density of resonators from the inner layer of the cloak to the outer layer, i.e. due to radial dispersion of air fractions in the shell. In addition, the requirement to avoid both coupling between resonators and strong resonance splitting as described in previous sections has been satisfied that has led to a spoke-type arrangement of the resonators in the cloak depicted in Fig. 10. The intent to exclude overlapping of the resonance fields "halos" limited minimal angular distances between spokes and minimal gaps between concentric arrays of resonators by the "halo" size, however, it did not deteriorate the cloak performance. The effective parameters of the cloak layers have been characterized by using mixing relations incorporating responses of air fractions and individual resonators (the latter were assigned not to the physical resonator bodies, but to the "halo" volumes). This approach allowed for accurate adjustment of the permeability dispersion, so that the desired performance of the cloak with no distortions of the wave front at frequencies within the cloaking band has been provided.
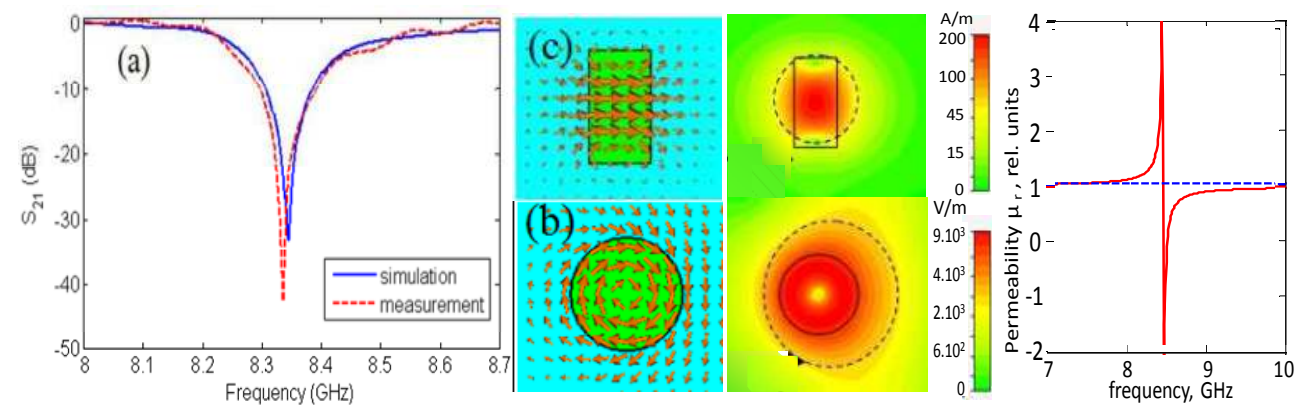

Fig. 9. (a) Simulated and measured $S_{12}$ spectra for a DR in WR137; (b) electric and (c) magnetic field patterns in DR cross-sections; and (c) effective permeability changes at the magnetic-type resonance in the DR (Chen et al., 2011). 


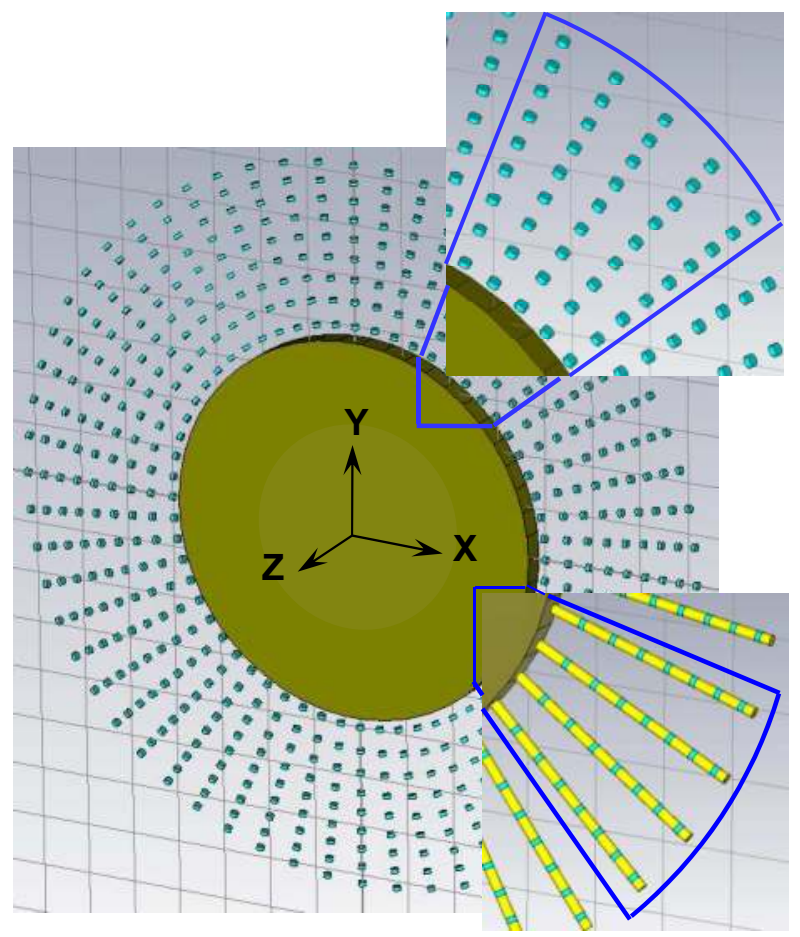

Fig. 10. The design of the infrared invisibility cloak composed of cylindrical glass resonators arranged in concentric arrays; lower inset shows the spokes of resonators with spacers (Semouchkina et al., 2010)

Despite the described advances the application of dielectric metamaterials in cloaking structures has underlined once again the main problem common for all resonance metamaterials - their narrowbandness. According to initial works on cloaking it was thought that application of such metamaterials excudes other than zero bandwidth for the invisibility effects (Pendry et al., 2006). Accordingly, the described above invisibility cloak demonstrated good cloaking effect in the band less than $1.2 \%$ or $3 \mathrm{THz}$. However, it was also revealed that even at a slightly denser packing of resonators than that shown in Fig. 11, the bandwidth could be increased up to 2 times. This effect could be related to the positive consequencies of coupling, which are known since earlier applications of resonator arrays in microwave filters and other devices, where coupling was used for the bandwidth enhancement. The next section will demonstrate the complex nature of coupling related phenomena in dielectric metamaterials and outline the opportunity to use them for the partial lifting of the delay-bandwidth limitations for cloaking devices formulated in (Hashemi et al., 2010).

\subsection{Specifics of coupling related phenomena in linear arrays of dielectric resonators}

The responses of linear arrays of DRs with the same parameters as those of the DR featured in Fig. 9, at their positioning along the wave propagation direction has been investigated first. In 
difference from close packed array of SRRs, even one pair of resonators placed at the distance between their bodies of $2 \mathrm{~mm}$ demonstrated resonance splitting of about $0.25 \mathrm{GHz}$ (Fig. 11a). Splitting decreased at increasing the distance between the resonators, however, it was still well expressed even at the separation of $10 \mathrm{~mm}$. Only $16 \mathrm{~mm}$ separation provided for an unsplit spectrum of $S_{21}$. It is worth mentioning that this distance is close to the doubled thickness of the electric field "halo" shown in Fig. 9. This confirms the suggestion that resonance coupling and splitting result from overlapping of "halos" created by neighboring resonators.
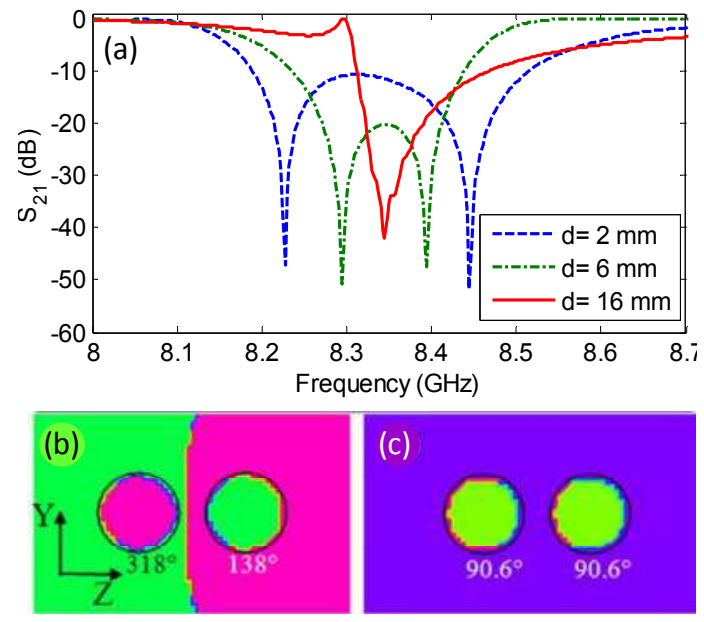

Fig. 11. (a) $S_{21}$ spectra for two DRs arranged along the axis of the waveguide WR137; (b) and (c) phase patterns for $\mathrm{H}$-field distributions in the longitudinal cross-section at 2 split resonances (Chen et al., 2011).

Phase patterns for magnetic field component in the waveguide cross-section presented in Fig. 11 show that at lower frequency, resonance field oscillations in two resonators proceed with opposite phases, while at higher frequency coherent resonance oscillations are observed. Since the resonance modes can be represented by magnetic dipoles, it follows that at lower frequency, magnetic dipoles formed in two resonators remain directed oppositely at any instant, while at higher frequency they always keep parallel orientation. Similar difference between split resonances at transverse interaction of resonators (side-by-side) was observed and discussed in (Liu et al., 2010), where it was concluded that dipole interaction defines the energy difference of two resonance states, and, correspondingly, the degree of their splitting in the frequency scale. In fact, parallel magnetic dipoles are expected to repel each other since the curls of their electric fields tend to cancell each other in the gap between the resonators. Just opposite, counter-directed magnetic dipoles provide for co-directed electric fields in the gap between the resonators that should attract them to each other, thus decreasing the state energy.

At increasing the quantity of resonators in the linear DR array from 2 to 5 , the split spectrum of $S_{21}$ demonstrated a better expressed gap of transmission in the frequency range of about $0.45 \mathrm{GHz}$ (Fig. 12). Further addition of resonators to the array led to increasing the quantity of transmission drops in the gap and to its gradual flattening. However, the signals of the probes that measured the magnitude of $\mathrm{H}$-field oscillations in the centers of resonators did 
not demonstrate any resonance-like field enhancement at corresponding frequencies even though they experienced deep drops of the resonance activity at some other frequencies. Most obvious resonance-like activity was seen only at frequencies corresponding to the boundaries of the transmission gap. Phase patterns in the waveguide cross-section at these frequencies reminded the patterns described above for the pair of resonators, i.e. at the low frequency boundary of the transmission gap all neighboring resonators oscillated with opposite phases, while at the higher frequency boundary all resonators in the array demonstrated coherent responses (Figs. 13a and 13b).
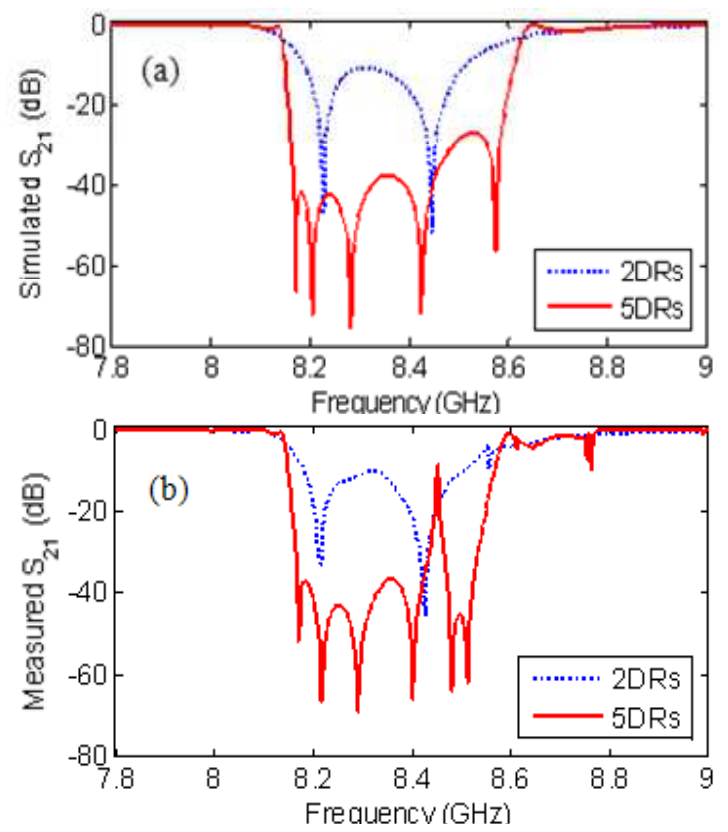

Fig. 12. (a) Simulated and (b) measured spectra of $S_{21}$ for linear DR arrays arranged along kvector at $2 \mathrm{~mm}$ between DR bodies (Chen et al., 2011).

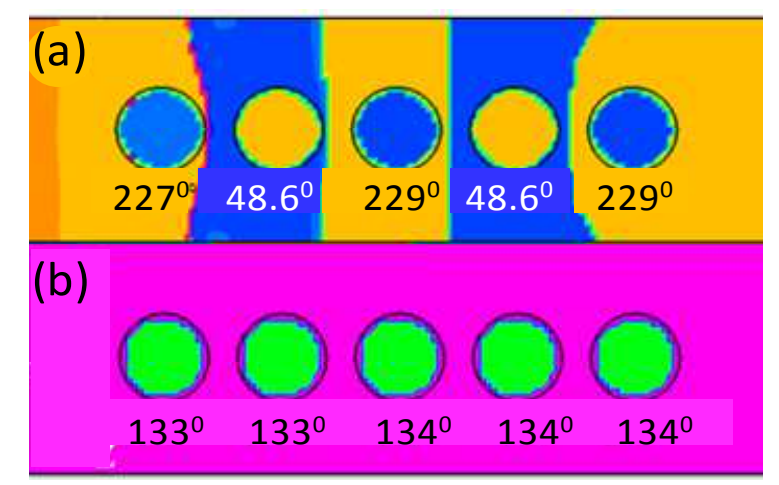

Fig. 13. H-field phase patterns in waveguide at (a) $8.17 \mathrm{GHz}$ and (b) $8.58 \mathrm{GHz}$ (Chen et al., 2011) 
It is worth noting that the revealed low frequency and high frequency resonance states in DR arrays remind bonding and anti-bonding states of electrons in molecules and crystals with such difference that instead of electron spins the type of resonance states in DR arrays is defined by respective orientations of formed magnetic dipoles. It is also important to add that a coherent response of resonators presents a special interest for all applications of metamaterials, which depend on the realization of the prescibed by the transformation optics dispersion of the effective medium parameters.

\subsection{EM responses of coupled 3D metamaterial arrays of dielectric resonators}

It should be pointed out that the described above simulations and experiments with placement the DR arrays in waveguides are, in fact, equivalent to modeling 3D arrays of resonators, due to the mirror reflection effect of the waveguide walls. Since the distance between resonators and the walls of the waveguide WR137 was relatively large compared to inter-resonator separation, the obtained results show that even large lattice parameters in two directions of the modelled 3D arrays (and, repectively, negligible coupling in these directions) does not provide the similarity of the array response to the response of the unit cell with a single resonator inside. However, large lattice parameters are rather unusual for metamaterials applications, therefore, the studies of dense 3D arrays that should present additional interest for practical realization of metamaterials, have also been conducted.

Numerical experiments with 3D arrays having lattice parameters between $8 \mathrm{~mm}$ and $12 \mathrm{~mm}$ (which corresponded to separations between the resonator bodies ranging from $2 \mathrm{~mm}$ to 6 $\mathrm{mm}$, respectively) were carried out in free space. Due to employment of periodic boundary conditions (PBC) in simulations, the 3D arrays under study were infinite in one direction and finite in two other directions. The plane wave incidence was normal to the infinite direction. For simplicity, a cubic structure of the arrays was employed. The individual responses of resonators in two perpendicular to each other linear arrays of resonators were controlled by the probes placed in the centers of corresponding resonators. In additition, more probes were placed at some distances from the array on the way of propagating waves to detect transmission (see schematic in Fig. 14a). Wave propagation through the arrays was controlled by using snapshots and animation of $\mathrm{H}$-field distribution patterns in $\mathrm{XZ}$ cross-section of the array.

As seen in Figs. 14b and 14c, the responses of array elements were characterized by multiple resonances observed even at the frequencies corresponding to the low frequecy half of the transmission gap, between $8.2 \mathrm{GHz}$ and $8.63 \mathrm{GHz}$ (see Fig. 14c). At frequencies below and above the transmission gap the waves passed through the array, however, the specifics of these propagations were quite different. While at lower frequencies the lengths of waves propagating in the array were essentially shorter than in free space so that the wave front movement through the array was lagging the wave front in free space (Fig. 15a), at higher frequencies, vise versa, the lengths of waves passing through the array were longer compared to the wavelengths in free space so that the movement of wave front through the array outrided the movement in free space (Fig. 15b). It is worth mentioning that when the frequency below the gap increased to approach its low frequency boundary, the lag in the wave movement through the array became more and more pronounced reaching its maximum at about $8.18 \mathrm{GHz}$. At frequencies above the transmission gap, the highest velocity of waves passing through the array was observed just near the gap boundary at about $8.66 \mathrm{GHz}$. At higher frequencies, wave advancing inside the array gradually decreased so that the entire wave front became flat at about $9.12-9.15 \mathrm{GHz}$. 

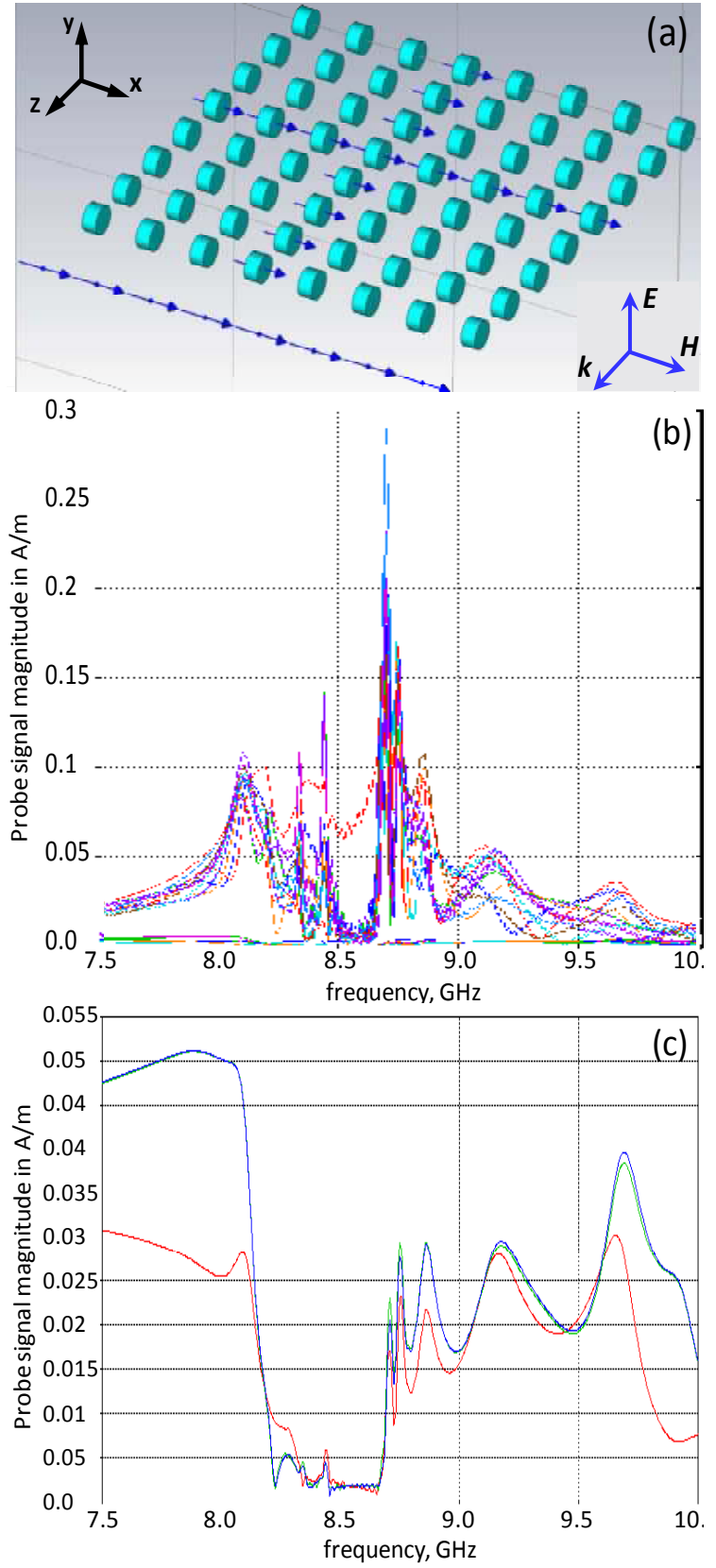

Fig. 14. (a) Basic element of 3D arrays at plane wave incidence in z-and PBC in y-direction; (b) spectra of signals from probes placed in DR centres; and (c) spectra of probes placed outside the sample to detect transmission. 

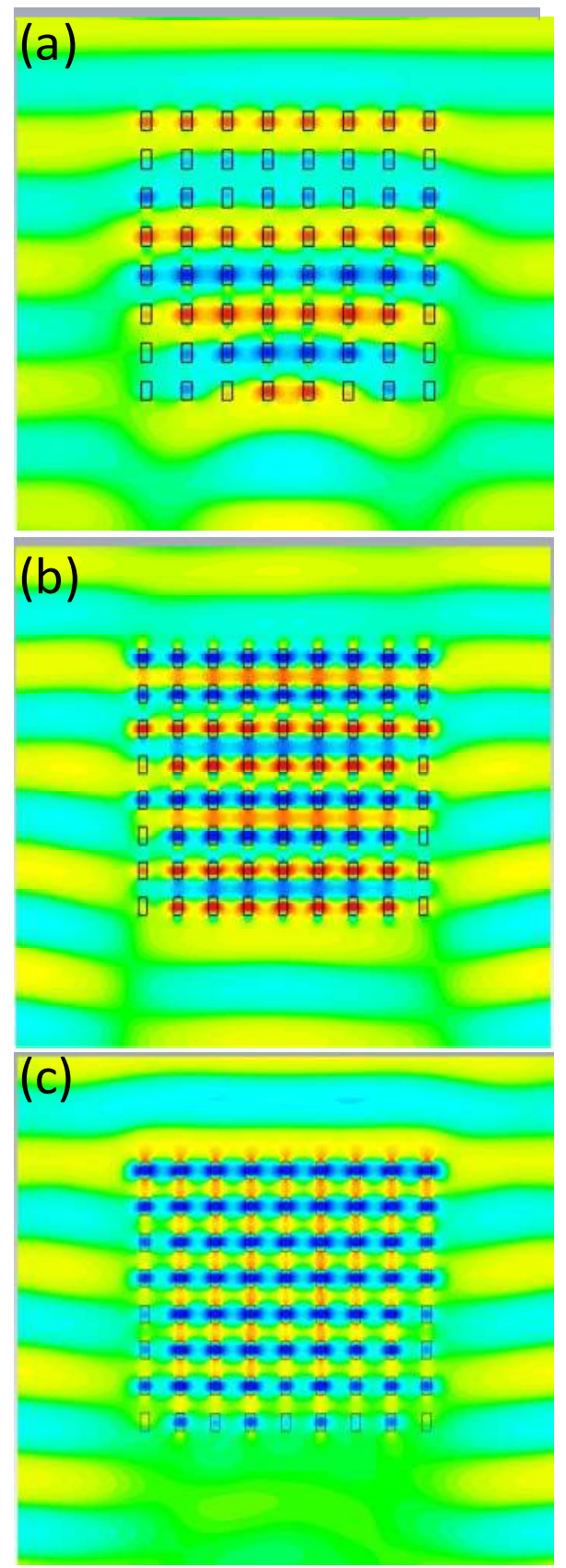

Fig. 15. Wave propagation in 3D DR arrays at frequencies (a) below the gap and (b, c) above the gap at: (a) $8.0 \mathrm{GHz}$; (b) $8.74 \mathrm{GHz}$; and (c) $8.64 \mathrm{GHz}$. 
If the investigated array could respond as a uniform medium without any resonance splitting it could be expected that the wave movement inside the array would depend on characteristic for Lorentz-type resonators changes of the effective permeability. As known, these changes have three essential for wave movement ranges: 1) increase of the effective permeability with frequency at approaching the resonance, 2) drop of the effective permeability at the resonance frequency down to negative values and then growth back with crossing the zero level at the so-called magnetic plasma frequency, and 3) continuing increase of the effective permeability in the range of values between 0 and 1 , as shown in Fig. 3c. The above changes would define the phase velocity of propagating waves in accordance with the known relation:

$$
v_{\mathrm{p}}=\frac{1}{\sqrt{\mu_{\mathrm{r}} \varepsilon_{\mathrm{r}}}}
$$

and cause lagging of the wave movement in the array at approaching the resonance, then a transmission gap at frequencies corresponding to the negative values of permeability, and, finally, superluminal wave movement at frequencies exceeding the magnetic plasma frequency. It seems doubtful to expect similar changes of the effective permeability as those observed in the model of a uniform medium (Fig. 3c) from close-packed arrays of coupled resonators with essentially split resonances. Nevertheless, the obtained data testify in favor of some analogy. It follows that despite of the resonance splitting, coupled arrays are capable of responding in the way predicted by the above described changes of the effective permeability near the resonance. It means that coupling and splitting of elementary resonances do not prevent arrays from specific integrated contributions of multiple responses to their effective properties and from exhibiting qualitatively similar to a uniform medium changes of these properties with frequency. Since application of metamaterials in cloaking devices assumes employment of cloaking shells for speeding up waves around the concealed objects, the obtained results show that these expectations could be realized despite of complications caused by the coupling phenomena. It is worth adding that at the "superluminal" phenomena, the resonators in coupled arrays tend to respond coherently within the half wavelengths of the passing waves. Therefore, at frequencies close to the upper boundary of the transmission gap, when the strongest extention of the wavelengths by the resonating array is provided, coherent response can involve even the entire array (Fig. 15c). It should be, however, taken into account that the coherent response formation could occur at frequencies different from the resonance frequency of a single resonator and that the frequency range of the coherent response could depend on the specifics of coupling effects in respective arrays.

The revealed possibility to employ arrays of coupled resonators for obtaining coherent responses within the half wavelengths of the incident wave provides the grounds for the employment of coupling phenomena in metamaterials to enhance the bandwidth of their operation and, in particular, for the enhancement of the cloaking effect bandwidth.

\section{Conclusion}

The performed studies of coupling and splitting of elementary resonances in arrays of metal split-ring resonators and dielectric resonators have demonstrated that ignoring coupling 
effects at metamaterial applications can lead to mistakes and malfunctioning. Even the best ideas proposed for designing various devices based on employing resonance metamaterials, can loose an opportunity to be realized properly unless coupling phenomena are taken into account. In particular, the presented data, which disclosed dramatic splitting of the resonances in close-packed arrays of SRRs have shown that these arrays could not be used for demonstrating the effects of invisibility by the cylindrical cloak of concentric resonator arrays at frequencies predicted byusing the analysis of a single SRR.

The arrays of dielectric resonators, which seem perspective due to low loss, especially for employment in cloaking devices, are also found to be a subject of coupling phenomena even at the lattice constants essentially exceeding the dimensions of resonators. However, there are reasons to express an optimism towards their applications as even at strong coupling and resonance splitting phenomena the DR arrays are able to demonstrate integrated responses qualitatively corresponding to the responses expected for the theoretical uniform media with Lorentz-type resonances. In particular, the DR arrays show an ability to support superluminal wave propagation and coherent resonance responses from multiple resonators located within the half wavelength of the propagating wave.

Similar to photonic crystals, DR arrays demonstrate the formation of a transmission gap that separates two transmission bands. However, this bandgap is not related to Bragg resonance and apparently is related to negative values of the effective permeability at elementary Lorentz-type resonances. In this attitude, metamaterial DR arrays could be compared to photonic crystals exploiting Mie resonances. The slow waves, which have been observed in DR arrays at frequencies below the transmission gap, and the superluminal waves observed at frequencies above the gap enhance the similarity with the latter photonic crystals. A detaled comparison of the properties and the physics underlying the observed phenomena in two types of artificial materials could provide a deeper understanding of their specifics.

\section{Acknowledgment}

This work was supported by the National Science Foundation under Grant No. 0968850. The author wishes to thank graduate students Fang Chen and Xiaohui Wang for performing some simulations and measurements.

\section{References}

Chen, F., Wang, X. \& Semouchkina, E. (2011). Simulation and Experimental Studies of Dielectric Resonator Arrays for Designing Metamaterials, Proceedings of IEEE International Symposium on Antennas and Propagation, pp. 2936-2939, ISSN: 15223965, Spokane, WA, August 2011

Cui, T. J., Smith, D. R., Liu, R. (2009), Metamaterials: Theory, Design, and Applications, Springer, 1st ed., (November 2009), ISBN-10: 1441905723

Gaillot, D. P., Croenne, C. \& L ippens, D. (2008). An All-dielectric Route for Terahertz Cloaking. Optics Express, Vol.16, No.6, (March 2008), pp. 3986-3992, ISSN: 1094-4087

Hashemi, H., Zhang, B., Joannopoulos, J. D. \& Johnson, S. G. (2010). Delay-Bandwidth and Delay-Loss Limitations for Cloaking of Large Objects. Phys. Rev. Lett., Vol.104, (June 2010), 253903, ISSN 0031-9007 
Jylha, L., Kolmakov, I., Maslovski, S., \& Tretyakov, S. (2006). Modeling of Isotropic Backward-Wave Materials Composed of Resonant Spheres. Journal of Applied Physics, Vol.99, 043102, (2006), ISSN:0021-8979

Kajfez, D. \& Guillon, P. (1998), Dielectric Resonators, Noble Publishing Corp., 2nd ed., Atlanta, (1998), ISBN:1884932053

Leonhardt, U. (2006). Optical Conformal Mapping. Science, Vol.312, No. 5781, (June 2006), pp. 1777-1779, ISSN 0036-8075

Liu, N. \& Giessen, H. (2010), Coupling Effects in Optical Metamaterials. Angewandte Chemie Int. Ed., Vol.49, No.51, (December 2010), pp. 9838-9852, DOI: 10.1002/anie.200906211, ISSN: 1521-3773

Pendry, J. B., Holden, A., Robbins, D. J, \& Stewart, W. J. (1999). Magnetism from Conductors and Enhanced Non-Linear Phenomena. IEEE Trans. on Microwave Theory $\mathcal{E}$ Techniques, Vol.47, No.11, (November 1999), pp. 2075-2084, ISSN: 0018-9480

Pendry, J. B. \& Smith, D. R. (2003). Reversing Light: Negative Refraction. Physics Today, Vol.56, (December 2003), pp. 1-8, ISSN:0031-9228

Pendry, J. B., Schurig, D. \& Smith, D. R. (2006). Controlling Electromagnetic Fields. Science, Vol.312, No.5781, (June 2006), pp. 1780-1782, ISSN 0036-8075

Schurig, D., Mock, J. J., Justice, B. J., Cummer, S. A., Pendry, J. B., Starr, A. F. \& Smith, D. R. (2006). Metamaterial Electromagnetic Cloak at Microwave Frequencies. Science, Vol.314, No.5806, (November 2006), pp. 977-979, ISSN 0036-8075

Semouchkina, E., Baker, A., Semouchkin, G., Randall, C. \& Lanagan, M. (2004). Resonant Wave Propagation in Periodic Dielectric Structures, Proceedings of the IASTED Int. Conf. ANTENNAS, RADAR AND WAVE PROPAGATION, pp. 149-154, ISBN: 088986-409-8, Banff, Canada, July 2004

Semouchkina, E., Semouchkin, G., Lanagan, M. \& Randall, C. A. (2005). FDTD Study of Resonance Processes in Metamaterials. IEEE Transactions on Microwave Theory $\mathcal{E}$ Techniques, Vol.53, (April 2005), pp. 1477-1487, ISSN: 0018-9480

Semouchkina, E., Werner, D. H. \& Pantano, C. (2009). An Optical Cloak Composed of Identical Chalcogenide Glass Resonators, Proceedings of the Metamaterials'2009 - $3^{\text {rd }}$ Int. Congress on Advanced Electromagnetic Materials in Microwaves and Optics, , ISBN 978-0-9551179-6-1, London, United Kingdom, September 2009

Semouchkina, E., Werner, D. H., Semouchkin, G. \& Pantano, C. (2010). An Infrared Invisibility Cloak Composed of Glass. Appl. Phys. Lett., Vol.96, 233503, (June 2010), ISSN 0003-6951

Smith, D. R., Pendry, J. B. \& Wiltshire, M. C. K. (2004). Metamaterials and Negative Refractive Index. Science, Vol.305, No.5685, (August 2004), pp. 788-792, ISSN 0036-8075

Smith, D. R. \& Pendry, J. B. (2006). Homogenization of Metamaterails by Field Averaging. JOSA B, Vol.B23, No.3, (March 2006), pp.391-403, ISSN: 0740-3224

Ueda, T., Lai, A. \& Itoh, T. (2007). Demonstration of Negative Refraction in a Cutoff ParallelPlate Waveguide Loaded with Two-Dimensional Lattice of Dielectric Resonators. IEEE Transactions on Microwave Theory \& Techniques, Vol.55, No.6, (June 2007), pp.1280-1287, ISSN: 0018-9480

Ueda, T., Michishita, N., Akiyama, M. \& and Itoh, T. (2010). Anisotropic 3-D Composite Right/Left-Handed Metamaterial Structures Using Dielectric Resonators and Conductive Mesh Plates. IEEE Transactions on Microwave Theory E Techniques, Vol.58, No.7, (July 2010), pp. 1766 -1773, ISSN: 0018-9480 
Vendik, O. G. \& M. S. Gashinova, M. S. (2004). Artificial Double Negative (DNG) Media Composed by Two Different Dielectric Sphere Lattices Embedded in a Dielectric Matrix, Proceedings of the 34th European Microwave Conference, pp. 1209-1212, ISBN/ISSN: 1580-5399-20 9781-5805-39920, Amsterdam, October 2004

Yariv, A., Xu, Y., Lee, R. K. \& Scherer, A. (1999).Coupled Resonator Optical Waveguide: a Proposal and Analysis. Optics Letters, Vol.24, No.11, (June 1999), pp.711-713, ISSN: 0146-9592

Zhang, B. L., Chen, H. S. \& Wu, B.-L. (2009). Practical Limitations of an Invisibility Cloak. Progress in Electromagnetics Research, Vol.97, (2009), pp. 407-416, ISSN: 1070-4698 


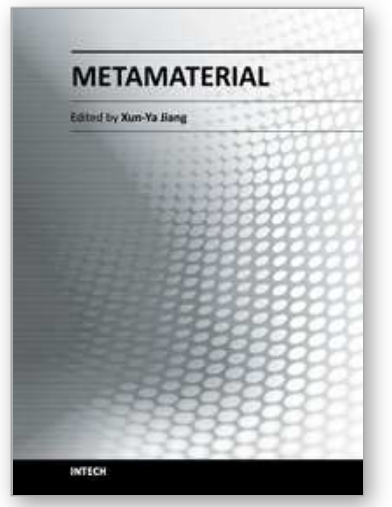

\author{
Metamaterial \\ Edited by Dr. Xun-Ya Jiang
}

ISBN 978-953-51-0591-6

Hard cover, 620 pages

Publisher InTech

Published online 16, May, 2012

Published in print edition May, 2012

In-depth analysis of the theory, properties and description of the most potential technological applications of metamaterials for the realization of novel devices such as subwavelength lenses, invisibility cloaks, dipole and reflector antennas, high frequency telecommunications, new designs of bandpass filters, absorbers and concentrators of EM waves etc. In order to create a new devices it is necessary to know the main electrodynamical characteristics of metamaterial structures on the basis of which the device is supposed to be created. The electromagnetic wave scattering surfaces built with metamaterials are primarily based on the ability of metamaterials to control the surrounded electromagnetic fields by varying their permeability and permittivity characteristics. The book covers some solutions for microwave wavelength scales as well as exploitation of nanoscale EM wavelength such as visible specter using recent advances of nanotechnology, for instance in the field of nanowires, nanopolymers, carbon nanotubes and graphene. Metamaterial is suitable for scholars from extremely large scientific domain and therefore given to engineers, scientists, graduates and other interested professionals from photonics to nanoscience and from material science to antenna engineering as a comprehensive reference on this artificial materials of tomorrow.

\title{
How to reference
}

In order to correctly reference this scholarly work, feel free to copy and paste the following:

Elena Semouchkina (2012). Formation of Coherent Multi-Element Resonance States in Metamaterials, Metamaterial, Dr. Xun-Ya Jiang (Ed.), ISBN: 978-953-51-0591-6, InTech, Available from: http://www.intechopen.com/books/metamaterial/formation-of-coherent-multi-element-resonance-states-inmetamaterials

\section{INTECH}

open science | open minds

InTech Europe

University Campus STeP Ri

Slavka Krautzeka 83/A

51000 Rijeka, Croatia

Phone: +385 (51) 770447

Fax: +385 (51) 686166

www.intechopen.com
InTech China

Unit 405, Office Block, Hotel Equatorial Shanghai

No.65, Yan An Road (West), Shanghai, 200040, China 中国上海市延安西路65号上海国际贵都大饭店办公楼 405 单元

Phone: +86-21-62489820

Fax: +86-21-62489821 
(C) 2012 The Author(s). Licensee IntechOpen. This is an open access article distributed under the terms of the Creative Commons Attribution 3.0 License, which permits unrestricted use, distribution, and reproduction in any medium, provided the original work is properly cited. 